\title{
Improved Iris Recognition in 2D Eigen Space
}

\author{
Abhijit Das \\ School of Education Technology \\ Jadavpur University \\ Kolkata, India
}

\author{
Ranjan Parekh \\ School of Education Technology \\ Jadavpur University \\ Kolkata India
}

\begin{abstract}
This paper a new biometric method for personal identification is been presented by iris identification of a person in lower dimensionality and reduced template size than the other previous approaches in 2D Eigen space, so that it can be use for verification in application areas. Here the iris images are expressed in lower dimension, re-tending its features by using covariance matrix and Eigen matrix to a covariant-Eigen space vector. The proposed approach is also suitable to work on half iris image. The proposed approach shows high accurate result.
\end{abstract}

\section{General Terms}

Pattern Recognition, Texture.

\section{Keywords}

Covariance matrix, Eigen matrix, Covariant-Eigen space

\section{INTRODUCTION}

Biometrics has gained its popularity as a technique for personal identification purposed extensively in the last decade due to its high reliability of the results obtained. Nowadays biometrics based security systems exist in many airports and other security checking places. Biometrics is a technique to authenticate or identify an individual, using physiological and behavioral characteristics like Iris scanning, Facial recognition, Fingerprint verification, Hand geometry, retinal scanning, Signature verification, Voice verification etc. Iris recognition is one of the most reliable and accepted methods for personal identification as it is difficult to duplicate. Iris is a highly protected internal organ of the eye, and iris patterns possess a high degree of randomness which enables generation of unique patterns for each individual, even identical twins. Moreover, the patterns remain stable throughout life and hence exhibit robustness.

Techniques for iris recognition involve a number of steps. The image is first captured from the physical world for processing, after which the iris position is identified in the captured image, by locating the outer and the inner boundaries of the iris. The iris region is subsequently segmented and separated from the original image. Features are then extracted from the segmented region which provides a mathematical model for comparison and identification.

One of the biggest challenges associated with iris recognition include building a reliable data model from random image patterns. Moreover it needs to be done in real time and in a way that do not consume large computational and storage resources.

The organization of the paper is as follows: section 2 provides an overview of related work, section 3 outlines the proposed methodology, section 4 provides details of the dataset and experimental results obtained, section 5 provides an analysis of the proposed work vis-à-vis earlier works and section 6 provides the overall conclusions and the scope for future research

\section{PAST APPROACHES}

In the early eighties of it was first discovered that iris pattern can be a way for personal verification as stated in [1]. John Daugman [2] was the first to propose a technique to identify a person using iris pattern. Daugman proposed an algorithm to segment the iris from the image of eye by integro-differential operators to detect the iris region faithfully and converting the circular iris image to rectangular shaped iris by dimension less pseudopolar coordinate system. In [3] the same segmentation and feature extraction process is used and the rectangular iris is converted to 2048 bit code. This creates a compact 256-byte template. In [4] the same algorithm of [3] is used to illustrate how iris detection works in Circuits and Systems for Video Technology and compared with a bigger dataset. In [5] the previous algorithm of [3] is modified for detecting and faithfully modeling the iris inner and outer boundaries with active contours, leading to more flexible embedded systems. In [6] a new technique for iris segmentation is proposed where it is demonstrated that only half portion of the iris is enough to detect a person. In [7] the author has used Eigen space for iris detection. In [8] DCT is used for feature extraction. In [9] scalar based Eigen space is used. In [10] Gabor filter is used for iris detection. In [11] the author proposes an iris recognition technique based on Zigzag Collarette Region and Asymmetrical Support Vector Machines. Paper [12] proposes an algorithm to localize the iris more quickly by improved Hough transform. In [13] Wavelet maxima moment in variant is used for iris feature extraction. A shape guided approach using game theory has been used for iris detection in [14]. In [15] a review of existing techniques on iris recognition is provided. Appearance-based periocular features in the context of face and non-ideal iris recognition is explained in [16]. In [17] as part of a video quality assessment module, a new local quality iris image method based on spectral energy analysis is proposed. In [18] the authors explain a Radial Symmetry Guided Particle Filter for Robust Iris Tracking. In [19] an Efficient Iris and Eye Corners Extraction Method have been proposed. In [20] a New Model of Securing Iris Authentication using Steganography is proposed.

\section{PROPOSED METHODLOGY}

\subsection{Image segmentation}

The preprocessing step involves a manual segmentation process for extraction of the iris textured portion from an eye image. An image processor has been used to demarcate and retain the relevant iris portion while discarding the other irrelevant portions of the eye. The extracted iris portion has 
been subsequently subjected to tonal correction using histogram equalization. Fig. 1 illustrates the basic steps.

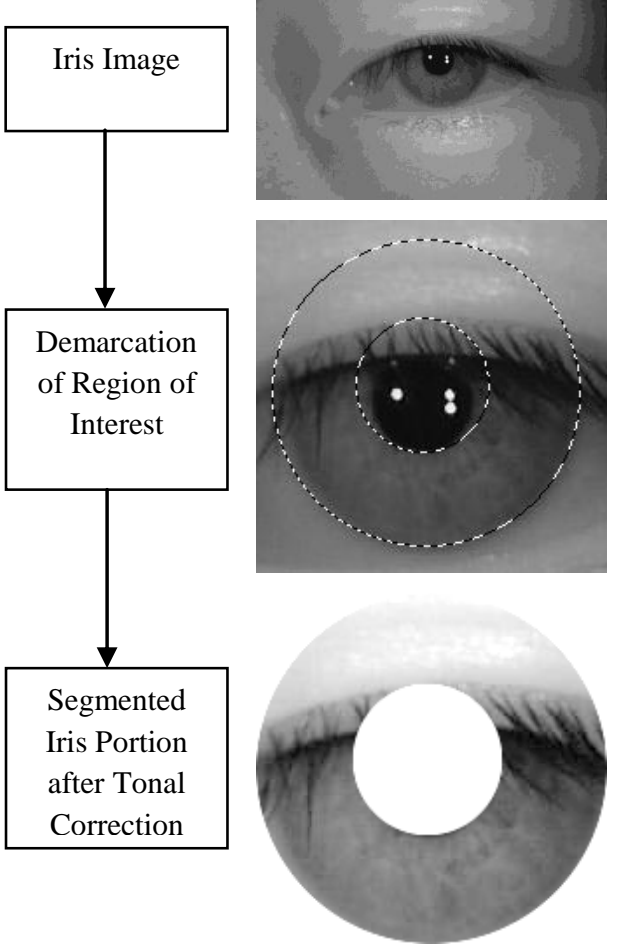

Fig 1: Steps of iris segmentation

\subsection{Feature Extraction Methodology}

In this algorithm the feature extraction process consist of two step first is mapping the iris image to Covariant-Eigen space i.e. calculating the Eigen vectors of the covariant matrix and second step calculating the weight scalar from the considered Eigen vector that is the feature of the algorithm for each iris

\subsubsection{Mapping images to Covariant-Eigen space}

Most of the previous works on iris detection, as noticed in [7] have used the issue of minimizing the aspect of the iris for identification and method of using a process to use only the variant feature or the main feature to reduce the feature vector size Here the segmented iris is first used to generate the covariance matrix which contains the main varying principal feature of the iris code. So the covariance matrix of the $i$-th person $j$-th sample is denoted by ${ }_{j}^{i} c$. Such ten ${ }_{j}^{i} c$ are averaged to get the average matrix $A_{i}$. Then $A_{i}$ is subtracted from each ${ }_{j}^{i} \mathrm{C}$ and transpose of the subtraction is multiplied to $\operatorname{get}_{j}^{i} M$.

$$
{ }_{j}^{i} M=\left({ }_{j}^{i} C-A_{i}\right) *\left({ }_{j}^{i} C-A_{i}\right)^{T}
$$

Then ten ${ }_{j}^{i} M$ are averaged to get ${ }^{i} M$ for the $i$-th person. From where the Eigen vector and the corresponding Eigen values are calculated. Only the Eigen vector derived from highest Eigen value, is consider for further calculation as in equation (2), the vector is denoted by ${ }^{i} v$.

$$
{ }^{i} Y={ }^{i} v^{\prime} *{ }^{i} M
$$

\subsubsection{Calculating weight scalar}

For calculating the weight we will calculate the iris vector by using equation (3) weight is denoted by $T_{i}$ which is multiplied with instance of the same person's image ${ }_{j}^{i} I$.

$$
T_{i}={ }_{j}^{i} I *{ }^{i} Y
$$

Then the two weight are average to get Hi Here Hi is the weight vector. For training and testing both we will follow the same equation for calculating scalar. During testing the scalar value is calculated in the same method and compared with the weight vectors of all the classes by absolute distance. The smallest distance is considered to be the class of the test image.

\section{EXPERIMENTAL RESULTS}

All simulation based experiments reported here are developed using the eye database is CASIA Iris Image Database [21]. We consider 20 classes and for each class we have 20 images To compute training feature vector we consider 10 images of each person for calculating the Eigen vector and 2 more for calculating the weight scalar i.e. a total of 12 images per class for training and the rest 8 for testing. Each of the images are $150 \times 150$ pixels in dimension and in JPG format

\subsection{Training phase}

In the training phase here we have consider 10 sample image of each person for calculating Eigen vector two more images for calculating weight i.e. all total 12 images for training. Few sample images used in the training are given in the figure 2.

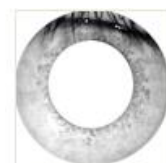

$1 \mathrm{~L} 01$

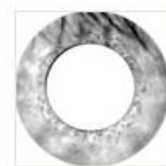

$2 \mathrm{LO}$

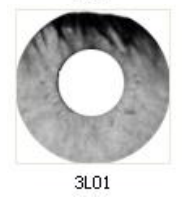

3L01

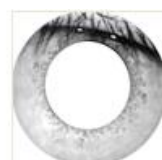

$1 \mathrm{L02}$

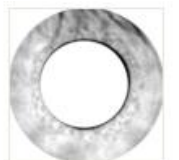

$2 \mathrm{~L} 02$

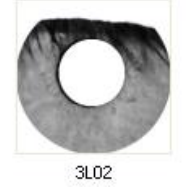

3L02

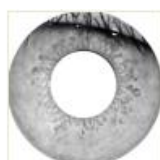

$1 \mathrm{L03}$

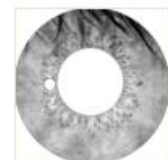

$2 \mathrm{~L} 03$

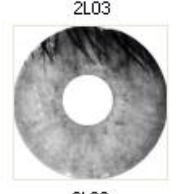

$3\llcorner 03$

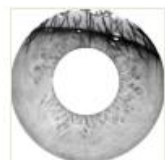

$1 \mathrm{LO4}$

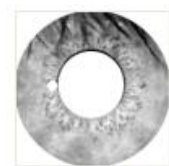

$2 \mathrm{~L} 04$

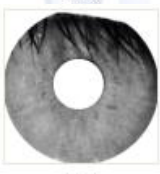

3L04
Fig 2: Training set images

The feature value plot for 20 classes is as shown in figure 3 .

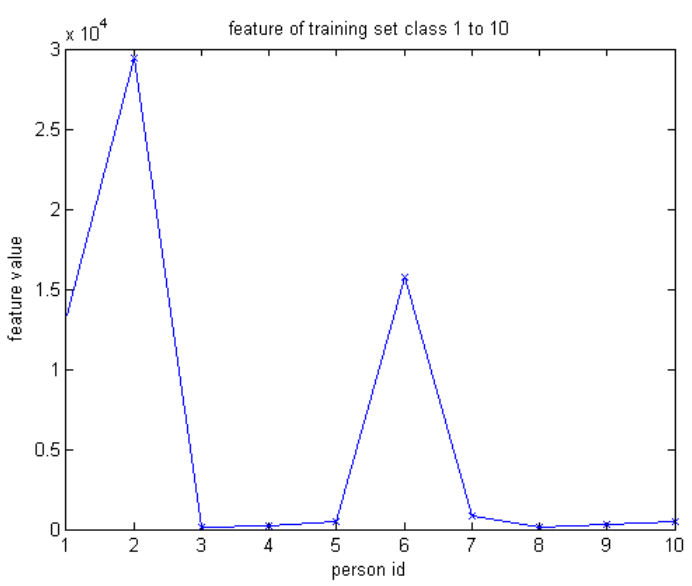




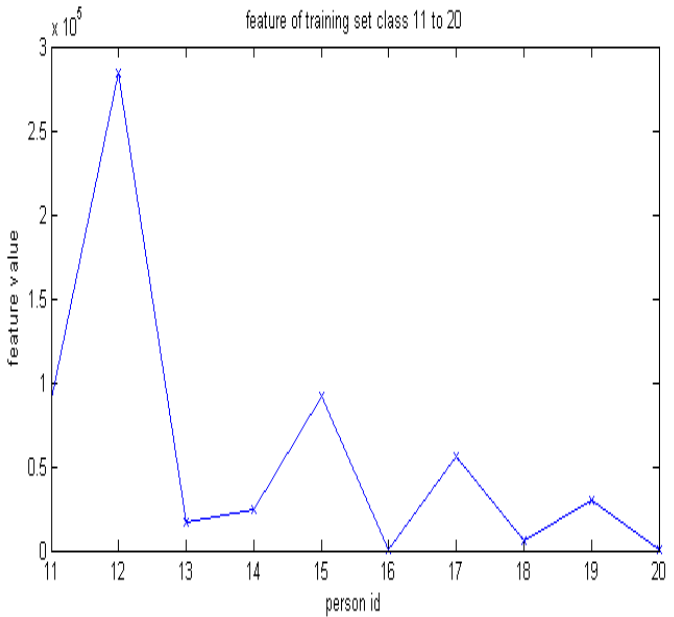

Fig 3: Feature plots of Training set

\subsection{Testing phase}

In the testing phase here we have consider eight sample image of each person. Few sample images used in the training are given in the fig 4.

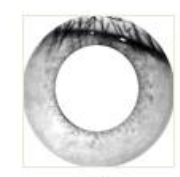

$1 L 17$

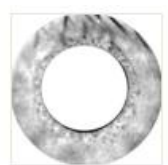

$2 \mathrm{~L} 17$

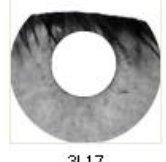

$3\llcorner 17$

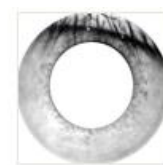

$1 \mathrm{~L} 18$

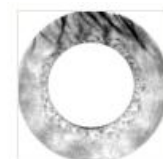

$2 \mathrm{~L} 18$

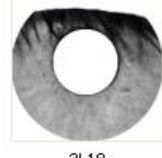

$3\llcorner 18$

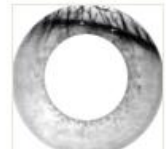

$1 L 19$

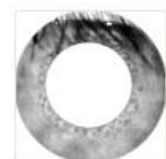

2L19

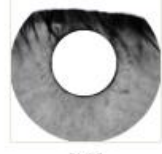

319

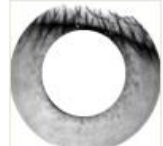

$1\llcorner 20$

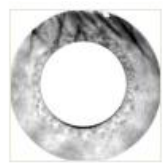

$2 \mathrm{~L} 20$

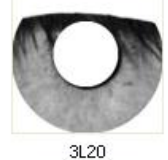

Fig 4: Testing set images

The feature value plots for 20 classes of iris images are shown in the figure 5 .

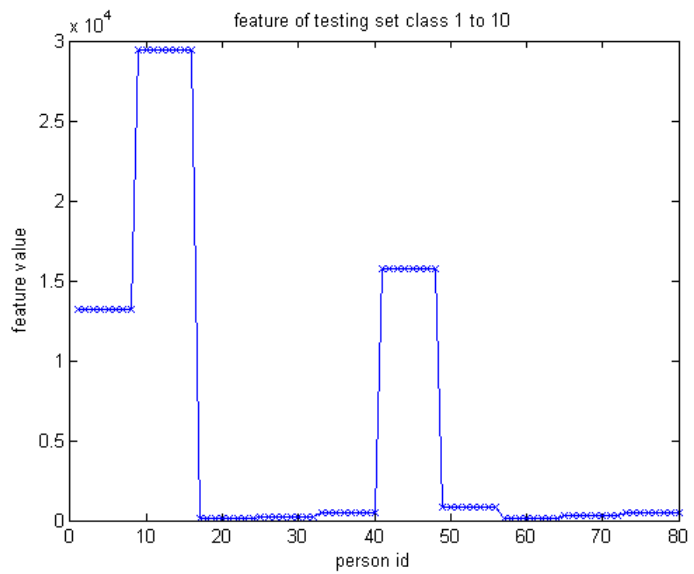

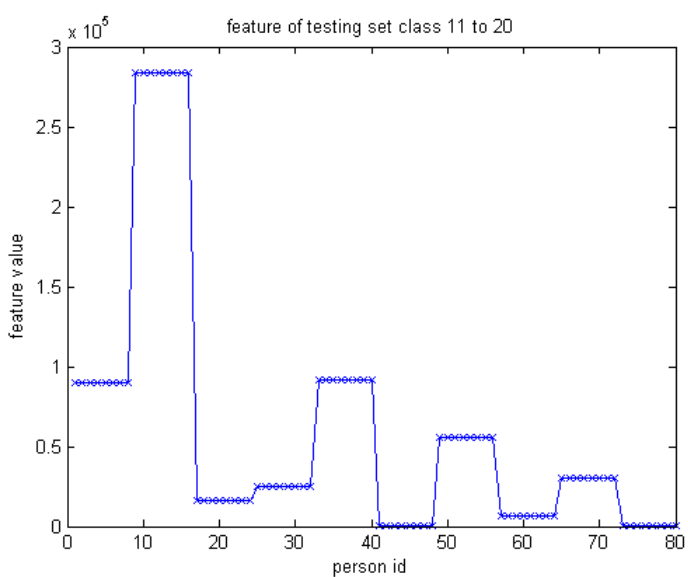

Fig 5: Feature plots of Testing set

\subsection{Computation of Estimated Classes}

The test images are compared with the training images to calculate the class by Scalar absolute distance. For each testing images by equation 3 scalars are calculated by multiplying ${ }^{i} Y$ of all the class. Respective absolute scalar difference is obtained between each scalar calculated and the previous training scalar of each class. The minimum absolute scalar difference is considered as the class of the particular image and the efficiency is found to be 100 percent. This can also be realized from the following diagram figure 6 .
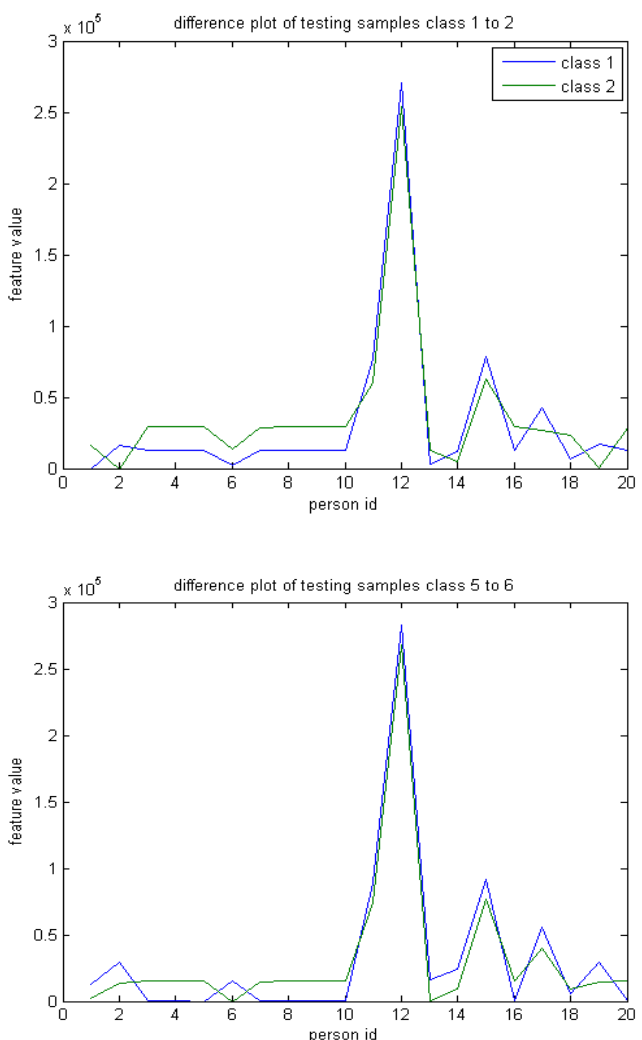

Fig 6: Class Estimation plots 


\section{ANALYSIS}

The recognition accuracy for discrimination the iris image of the each class is done successfully and with satisfactory accuracy. To put the results in perspective with the state-ofthe-art, we analyzed the most similar work we could find i.e. [7] with regards to recognition accuracy tested on the same dataset. In [7] out of 15 iris images of each person, 10 are used for training set, 2 for calculating the weight vector and the remaining 3 for testing. The first 10 image matrices are converted from 2-D to 1-D column vectors $x_{1}, \ldots, x_{10}$ and concatenated together to form a $\left(n^{2} \times 10\right)$ matrix where $(n \times n)$ is the dimension of each image. If $X$ is the concatenated matrix for a single person or class, we have

$$
X=\left[x_{1}, x_{2}, x_{3}, x_{4}, x_{5}, x_{6}, x_{7}, x_{8}, x_{9}, x_{10}\right]=\bigcup_{k=1}^{10} x_{k}
$$

The row-wise mean is calculated and represented by $M$ $\left(n^{2} \times 1\right)$. The mean is subtracted from each of the 1-D column matrices to generate normalized matrices. The normalized matrices are again concatenated side by side to form $D\left(n^{2} \times\right.$ 10)

$$
D=\bigcup_{k=1}^{10} \varphi_{k}, \text { where }, \varphi_{k}=x_{k}-M
$$

A $(10 \times 10)$ square matrix $E$ is generated by multiplying $D$ with its transpose, and eigenvectors $E_{C}$ and eigenvalues $E_{L}$ of $E$ are generated. A vector $U\left(n^{2} \times 10\right)$ is formed by product of $D\left(n^{2} \times 10\right)$ and $E_{C}(10 \times 10)$

$$
U=D \cdot E_{C}
$$

Weight vectors $w_{1}$ and $w_{2}$, each of dimension $(10 \times 1)$, are calculated from the second set of 2 images and these are averaged to form an average weight $W(10 \times 1)$ vector for a class :

$$
\begin{aligned}
w_{1} & =U^{T} \cdot\left(x_{11}-M\right) \\
w_{2} & =U^{T} \cdot\left(x_{12}-M\right) \\
W & =\left(w_{1}+w_{2}\right) / 2
\end{aligned}
$$

Testing is done using the remaining 3 images for each class by calculating their individual weight vectors:

$$
\begin{aligned}
& w_{3}=U^{T} .\left(x_{13}-M\right) \\
& w_{4}=U^{T} .\left(x_{14}-M\right) \\
& w_{5}=U^{T} .\left(x_{15}-M\right)
\end{aligned}
$$

The $j$-th test vector $w_{j}$ is compared with each of the 10 training vectors $W_{1} \ldots W_{10}$ using Euclidean distance. Let the distance values be $D_{j, 1} \ldots D_{j, 10}$. A test image is classified to the class with which its Euclidean distance is minimum.

$$
w_{j} \rightarrow k \text { if } \min \left(D_{j .1}, \ldots, D_{j .10}\right)=D_{j, k}
$$

The above scheme when applied to the current dataset produces training weight vectors for the 10 classes as shown below in Fig. 7
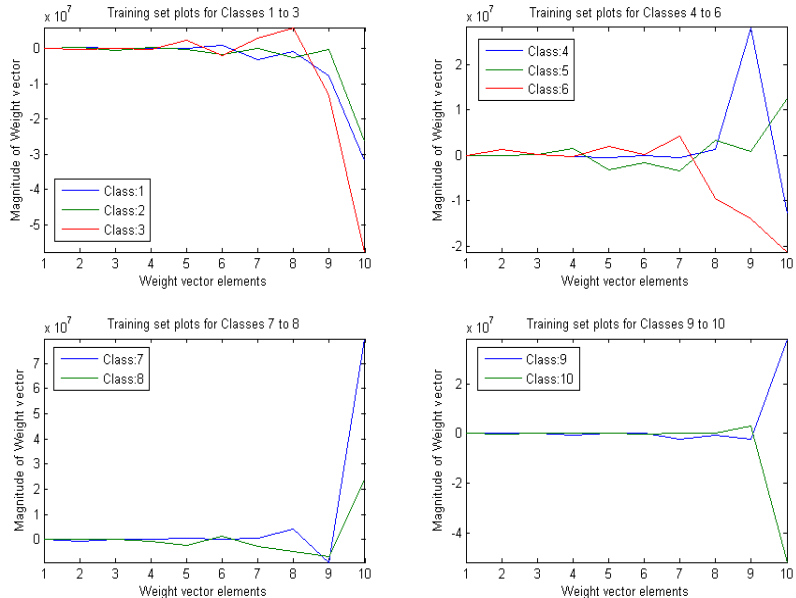

Fig 7: Feature plots for previous work [7]

The following figure Fig. 8 depicts the difference of the test samples of the first four classes with the training set of all classes.
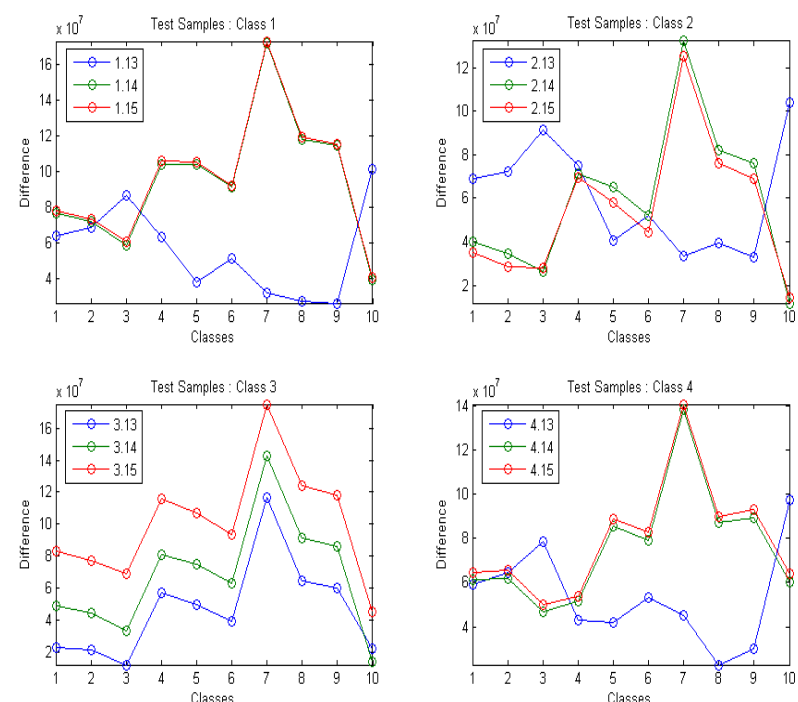

Fig 8: Difference plots for previous work [7]

The accuracy of the previous algorithm in [7] on the current dataset is observed to be $40 \%$ when 3 test samples per class are considered and $38.75 \%$ when 8 test samples per class are considered. In both cases a 10-element feature vector is used and comparisons are done using Euclidean distance. In contrast, the current work achieves an accuracy of $100 \%$ with 8 samples per class, using scalar feature values, compared using simple scalar differences. A comparison chart is shown in Table 1.Another previous work in [9] the author presents another work of iris recognition on Eigen plane. The feature plots of the work's test sample are as following. 


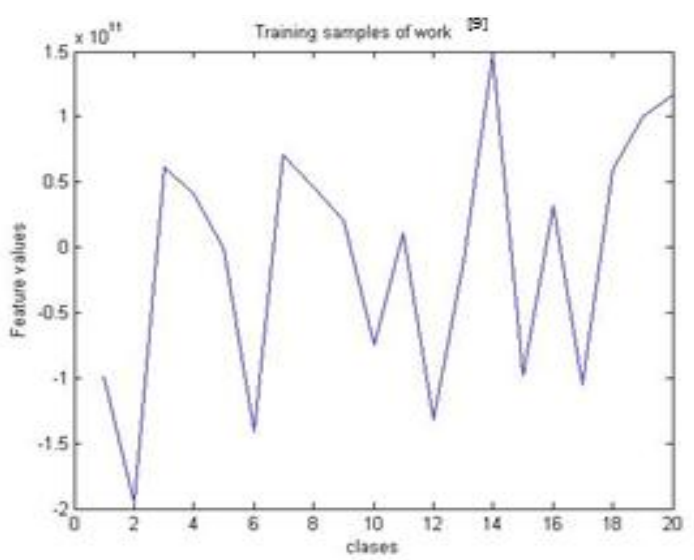

Fig 9: Feature plots for previous work [9]

The following figure Fig. 8 depicts the difference of the test samples of the first two classes with the training set of all classes.
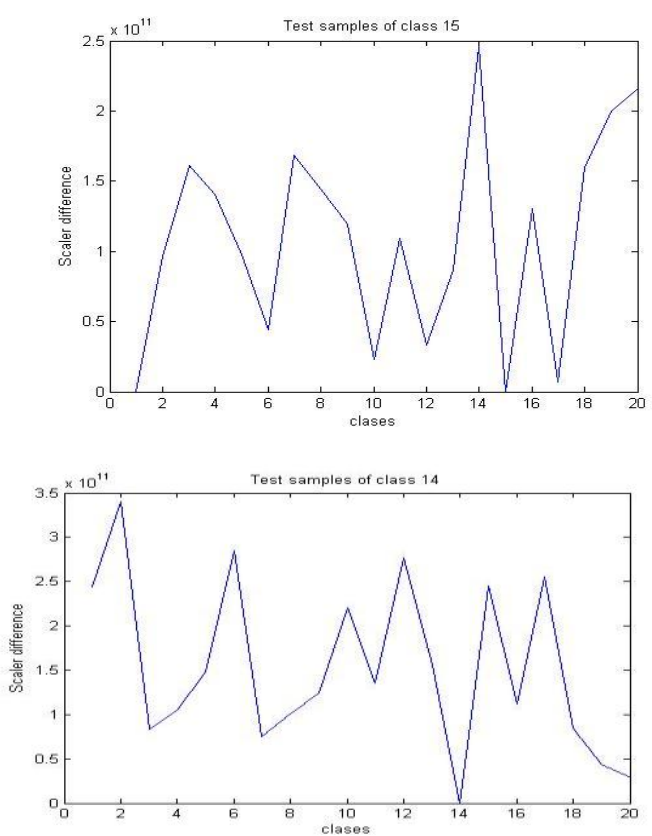

Fig 10: Difference plots for previous work [9]

The recognition accuracy for discrimination the iris image of the each class is done successfully and with satisfactory accuracy. The accuracy comparison of the previous algorithm in [7] \& [9] and the proposed algorithm is as following in table 1 .

Table 1. Comparison of Recognition Accuracies

\begin{tabular}{|c|c|c|c|c|}
\hline $\begin{array}{c}\text { Approa- } \\
\text { ches }\end{array}$ & $\begin{array}{c}\text { Feature } \\
\text { represen- } \\
\text { tation }\end{array}$ & Comparison & $\begin{array}{c}\text { Accuracy } \\
\text { (3 test } \\
\text { samples) }\end{array}$ & $\begin{array}{c}\text { Accuracy } \\
\text { (8 test } \\
\text { samples) }\end{array}$ \\
\hline $\begin{array}{c}\text { Previous } \\
\text { Work [7] }\end{array}$ & $\begin{array}{c}10- \\
\text { element } \\
\text { vector }\end{array}$ & $\begin{array}{c}\text { Euclidean } \\
\text { distance }\end{array}$ & $38 \%$ & $36 \%$ \\
\hline $\begin{array}{c}\text { Previous } \\
\text { Work [9] }\end{array}$ & $\begin{array}{c}\text { 1-element } \\
\text { scalar }\end{array}$ & $\begin{array}{c}\text { Scalar } \\
\text { difference }\end{array}$ & $100 \%$ & $96 \%$ \\
\hline $\begin{array}{c}\text { Current } \\
\text { Work }\end{array}$ & $\begin{array}{c}\text { 1-element } \\
\text { scalar }\end{array}$ & $\begin{array}{c}\text { Scalar } \\
\text { difference }\end{array}$ & $100 \%$ & $100 \%$ \\
\hline
\end{tabular}

\section{CONCLUSIONS \& FUTURE SCOPES}

The proposed approach has been proved to be an accurate and optimized technique providing better recognition accuracies and smaller feature representations than contemporary works in extant literature. Salient features of the proposed technique include a low dimensional feature representation of the iris texture, low computational overheads involving only scalar arithmetic and high recognition accuracies. This enables recognition and identification possible in real-time applications. Another important characteristic of this work is the robustness of the method which provides satisfactory results even in presence of eyelids and eyelashes which partially occludes the iris portion. In this work a manual preprocessing step was used to isolate and segment the iris portion from the rest of the eye. Future work would involve an automated segmentation technique involving an edge detector for detecting the iris area from eye images.

\section{REFERENCES}

[1] Bertillon, A, 1885. La couleur de Piris. Revue scientifique. Vol. 36, No. 3, 65-73.

[2] Daugman, J. 1992. High confidence personal identification by rapid video analysis of iris Texture. Proc. IEEE International Carnaha conf. on security technology, 50-60.

[3] Daugman, J. 1993. High confidence visual recognition of persons by a test of statistical independence. IEEE Trans. on Pattern Analysis and Machine Intelligence, Vol.15, No. 11, 1148-1161.

[4] Daugman, J. 2004. How iris recognition works. Circuits and Systems for Video Technology. IEEE Trans., Vol. 14, No. 1, 21-30.

[5] Daugman, J. 2007. New Methods in Iris Recognition. Proc. IEEE Trans. on system, man and cybernetics-part B, Vol. 37, No 5, 1167-1175.

[6] Savithiri, G., and Murugan, A. 2011. Performance Analysis on Half Iris Feature Extraction using GW, LBP and HOG. International Journal of Computer Applications, Vol. 22, No.2 (May 2011), 27-32.

[7] Hussain, Md. A. 2010. Eigenspace Based Accurate Iris Recognition System. Proc. Annual IEEE India Conference (INDICON), 763-767.

[8] Monro, D. M., Rakshit, S., and Zhang, D. 2007. DCTBased Iris Recognition. IEEE Trans. Pattern Analysis and Machine Intelligence, Vol. 29, No. 3 (April 2007), 586-595.

[9] Das, A., and Parekh, R. 2012. Iris Recognition using a Scalar base Template in Eigen-space. IJCST, Vol.3, No.5, 74-79.

[10] Wang, Q, Zhang, X., Li, M., Dong, X., Zhou, Q., and Yin, Y. 2012. Adaboost and multi-orientation 2D Gaborbased noisy iris recognition. Pattern Recognition Letters, Vol. 33, 978-983.

[11] Roy, K., and Bhattacharya, P. 2007. Iris Recognition Based on Zigzag Collarette Region and Asymmetrical Support Vector Machines. International Conference on Image Analysis and Recognition, 854-865. 
[12] Wang, L., Yang, G., and Yin, Y. 2010. Fast Iris Localization Based on Improved Hough Transform. Rough Set and Knowledge Technology, 439-446.

[13] Nabti, M. and Bouridane, A. 2007. An Improved Iris Recognition System Using Feature Extraction Based on Wavelet Maxima Moment Invariants. International Conference on Biometrics, 988 - 996.

[14] Roy, K., Bhattacharya, P., and Ching, Y. S. 2011. Iris recognition using shape-guided approach and game theory. Pattern Anal Application, Vol. 14, 329-348.

[15] Radman, A., Jumari, K., and Zainal, N. 2011. Iris Segmentation: A Review and Research Issues. International Conference on Software Engineering and Computer Systems, 698-708.

[16] Woodard, D.L., Pundlik, S.J., Miller,P.E., and Lyle, J.R. 2011. Appearance-based periocular features in the context of face and non-ideal iris recognition. Signal, Image and Video Processing, Vol.5, 443-455.
[17] Luis Miguel Zamudio-Fuentes1, Mireya S. GarcíaVázquez, and Alejandro Alvaro Ramírez-Acosta 2011. Local Quality Method for the Iris Image Pattern. Computer Analysis of Images and Patterns, 79-88.

[18] Martinez, F., Carbone, A., and Pissaloux, E. 2011. Radial Symmetry Guided Particle Filter for Robust Iris Tracking. Computer Analysis of Images and Patterns, 531-539.

[19] Erdogmus, N., and Dugelay, J. 2010. An Efficient Iris and Eye Corners Extraction Method. Structural, Syntactic, and Statistical Pattern Recognition, 549-558.

[20] Abidin, Z.Z., Manaf, M., and Shibghatullah, A.S. 2011. A New Model of Securing Iris Authentication Using Steganography. International Conference on Software Engineering and Computer Systems, 547-554.

[21] CASIA Iris Image Database Version 3.0 (CASIAIrisV3), Available: www.biometrics.idealtest.org 